hep-ph 9403361

SLAC-PUB-6464

Revised Version

May 1994

\title{
ANGULAR DISTRIBUTIONS IN THE DRELL-YAN PROCESS: A CLOSER LOOK AT HIGHER TWIST EFFECTS
}

\author{
A. Brandenburg ${ }^{1,2}$, S. J. Brodsky², V. V. Khoze ${ }^{2}$ and D. Müller ${ }^{2,3}$ \\ Stanford Linear Accelerator Center \\ Stanford University, Stanford, California 94309
}

\begin{abstract}
We calculate the angular distribution of the lepton produced in the Drell-Yan reaction taking into account pion bound state effects. We work in the kinematic region where one of the pion constituents goes far off-shell, which allows us to treat the bound state problem perturbatively. We show that the angular distribution is very sensitive to the shape of the pion distribution amplitude. The model we discuss fits the data if we choose a two-humped pion distribution amplitude suggested by QCD sum rules.
\end{abstract}

Submitted to Phys. Rev. Let.

\footnotetext{
1 Max Kade fellow

2 Work supported by the Department of Energy, contract DE-AC03-76SF00515

3 Supported by Deutscher Akademischer Austauschdienst
} 
Lepton pair production in hadron-hadron collisions provides a basic testing ground for our understanding of strong interactions. Extensive experimental and theoretical work has been done in the past two decades (for reviews see Ref. [1]). In particular, the angular distribution of the lepton pair has been studied in detail, revealing a fatal disagreement of the QCD improved parton model prediction [2,3] with the data [4-6]. Recently it has been proposed that this problem may be resolved if the nontrivial structure of the QCD vacuum induces spin correlations between the initial state partons [7]. In this letter we pursue another way to go beyond the standard parton model picture, namely we consider contributions to the angular distribution induced by hadron bound state effects. Our approach is close in spirit to the higher twist model of $[8,9]$.

The angular distribution of the $\mu^{+}$in

$$
\pi^{-}+N \rightarrow \gamma^{*}+X \rightarrow \mu^{+}+\mu^{-}+X
$$

may be parameterized in general as follows:

$$
\frac{1}{\sigma} \frac{d \sigma}{d \Omega} \sim 1+\lambda \cos ^{2} \theta+\mu \sin 2 \theta \cos \phi+\frac{\nu}{2} \sin ^{2} \theta \cos 2 \phi
$$

Here $\theta$ and $\phi$ are angles defined in the muon pair rest frame and $\lambda, \mu$ and $\nu$ are angleindependent coefficients. The naive parton model (Drell-Yan picture [10]) views the production of the virtual photon $\gamma^{*}$ in (1) as originating from the annihilation of two uncorrelated constituent quarks, resulting in an angular distribution of the form $1+\cos ^{2} \theta$. This result follows simply from the fact that the virtual photon is produced transversely polarized in the annihilation of two on-shell fermions.

In order to describe the boson transverse momentum distribution $d^{2} \sigma / d Q_{T}^{2}$ one has to take into account radiative corrections to the Drell-Yan model. The $Q_{T}$-distribution has been calculated in the QCD-improved parton model to the order of $\mathcal{O}\left(\alpha_{s}\right)$ with resummation of the soft gluons at the leading double logarithmic accuracy (see Ref. [11] and references therein). This approach was used in [3] to compute the angular distribution at fixed transverse momentum. The deviations from the $1+\cos ^{2} \theta$ behavior were found to be less than $5 \%$ in the range $0<Q_{T}<3 \mathrm{GeV}[3]$. However, the NA-10 measurements from CERN [4] and the Chicago-Iowa-Princeton collaboration [5,6] show a quite different behavior. In the limit where the momentum fraction $x$ of one of the pion constituents is 
very close to 1 and for moderate transverse momenta of the muon pair, the value of $\lambda$

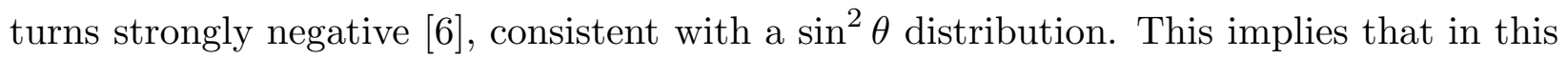
kinematic limit the virtual photon is produced with longitudinal polarization, rather than transverse. Furthermore, the data [4-6] is observed to have a strong azimuthal modulation (nonzero $\mu$ and $\nu$ in (2)), an effect which is missing in standard QCD. The Lam-Tung sum rule [2], $1-\lambda-2 \nu=0$, which follows from the approach used in [3] is also badly violated by the experimental data.

One way to go beyond the standard treatment is to take into account the pion bound state effects $[8,9]$. We want to treat the bound state problem perturbatively; thus we will restrict ourselves to a specific kinematic region in which the momentum fraction $x$ of one of the pion constituents is large, $x>0.5$. In fact, in the large $x$ region the off-shell nature of the annihilating quark from the projectile is crucial, and thus the operative subprocess must involve the correlated multi-parton structure of the projectile. In effect the dominant subprocess in the off-shell domain is $\pi^{-} q \rightarrow \mu^{+} \mu^{-} q$. We resolve the pion by a single hard gluon exchange [12]. The main contribution to reaction (1) then comes from the diagrams of Fig. 1a,b $[8,9]$. We see from diagram 1a that the $\bar{u}$ quark propagator is far off-shell, $p_{\bar{u}}^{2}=-Q_{T}^{2} /\left(1-x_{\bar{u}}\right)$. The second diagram is required by gauge invariance ${ }^{\dagger}$. The leading contribution to the amplitude $M$ for the reaction

$$
u+\pi^{-} \rightarrow \gamma^{*}+X \rightarrow \mu^{+}+\mu^{-}+X
$$

is obtained [12] by convoluting the partonic amplitude $T\left(u+\bar{u} d \rightarrow \gamma^{*}+d \rightarrow \mu^{+}+\mu^{-}+d\right)$ with the pion distribution amplitude $\phi\left(z, \tilde{Q}^{2}\right)$,

$$
M=\int_{0}^{1} d z \phi\left(z, \tilde{Q}^{2}\right) T
$$

where $\tilde{Q}^{2} \sim Q_{T}^{2} /(1-x)$ is the cutoff for the integration over soft momenta in the definition of $\phi$. In the regime where $\tilde{Q}^{2}$ and $Q^{2}$ are compatible, one cannot use the usual probabilistic factorization of the structure functions and the hard annihilation subprocess [13].

For the hadronic differential cross section we have

$\dagger$ In a physical gauge the contribution of the second diagram is purely higher twist, that is it contains extra powers of $\sqrt{Q_{T}^{2} / Q^{2}}$. 


$$
\begin{gathered}
\frac{Q^{2} d \sigma\left(\pi^{-} N \rightarrow \mu^{+} \mu^{-} X\right)}{d Q^{2} d Q_{T}^{2} d x_{L} d \Omega}=\frac{1}{(2 \pi)^{4}} \frac{1}{64} \int_{0}^{1} d x_{u} G_{u / N}\left(x_{u}\right) \int_{0}^{1} d x_{\bar{u}} \frac{x_{\bar{u}}}{1-x_{\bar{u}}+Q_{T}^{2} / Q^{2}}|M|^{2} \\
\delta\left(x_{L}-x_{\bar{u}}+x_{u}-Q_{T}^{2} s^{-1}\left(1-x_{\bar{u}}\right)^{-1}\right) \delta\left(Q^{2}-s x_{u} x_{\bar{u}}+Q_{T}^{2}\left(1-x_{\bar{u}}\right)^{-1}\right)+\{u \rightarrow \bar{d}, \bar{u} \rightarrow d\} .
\end{gathered}
$$

Here $Q^{\mu}$ is the four-momentum of $\gamma^{*}$ in the hadronic center of mass system, $x_{u(\bar{u})}$ is the light-cone momentum fraction of the $u(\bar{u})$ quark and $G_{u / N}$ is the parton distribution function of the nucleon. The longitudinal momentum fraction of the photon is defined as $x_{L}=2 Q_{L} / \sqrt{s}$ and it should be noted that its maximum value, $x_{L}^{\max }=1-s^{-1}\left(Q^{2}+2 Q_{T}^{2}\right)$ is slightly less than 1 . The second term on the right hand side of (5) is the same as the first one with quark flavors interchanged. This term gives the contribution from the nucleon sea. In Fig. 1c we show a typical contribution to the hadronic cross section.

We note that no primordial or intrinsic transverse momenta have been introduced. The single gluon exchange is the only source of $Q_{T}$ in the model discussed. We also neglected the quark masses and the mass of the projectile which are small compared to $\tilde{Q}$.

In analogy to eq. (2) we parameterize the angular distribution as follows,

$$
\begin{gathered}
\frac{Q^{2} d \sigma}{d Q^{2} d Q_{T}^{2} d x_{L} d \Omega}\left(\frac{Q^{2} d \sigma}{d Q^{2} d Q_{T}^{2} d x_{L}}\right)^{-1}= \\
\frac{3}{4 \pi} \frac{1}{\lambda+3}\left(1+\lambda \cos ^{2} \theta+\mu \sin 2 \theta \cos \phi+\frac{\nu}{2} \sin ^{2} \theta \cos 2 \phi\right),
\end{gathered}
$$

where the angular distribution coefficients $\lambda, \mu$ and $\nu$ are now functions of the kinematic variables $x_{L}, Q_{T}^{2} / Q^{2}$ and $Q^{2} / s$.

We work in the Gottfried-Jackson frame where the $\hat{z}$ axis is taken to be the pion direction in the muon pair rest frame and the $\hat{y}$ axis is orthogonal to the $\pi^{-} N$ plane. With some algebra, using eqs. (4)-(6), we arrive at the following expression for $\lambda, \mu$ and $\nu$,

$$
\begin{gathered}
\lambda\left(\tilde{x}, Q_{T}^{2} / Q^{2}\right)=2 N^{-1}\left\{(1-\tilde{x})^{2}\left[(\operatorname{Im} I(\tilde{x}))^{2}+(F+\operatorname{Re} I(\tilde{x}))^{2}\right]\right. \\
\left.-4 Q_{T}^{2} / Q^{2} \tilde{x}^{2} F^{2}+Q_{T}^{4} / Q^{4} \tilde{x}^{2} F^{2}\right\}, \\
\mu\left(\tilde{x}, Q_{T}^{2} / Q^{2}\right)=-4 N^{-1} \sqrt{Q_{T}^{2} / Q^{2}} F \tilde{x}\left\{(1-\tilde{x})[F+\operatorname{Re} I(\tilde{x})]+Q_{T}^{2} / Q^{2} \tilde{x} F\right\},
\end{gathered}
$$




$$
\nu\left(\tilde{x}, Q_{T}^{2} / Q^{2}\right)=-8 N^{-1} Q_{T}^{2} / Q^{2} \tilde{x}(1-\tilde{x}) F[F+\operatorname{Re} I(\tilde{x})]
$$

where

$$
\begin{gathered}
F=\int_{0}^{1} d z \frac{\phi\left(z, \tilde{Q}^{2}\right)}{z} \\
I(\tilde{x})=\int_{0}^{1} d z \frac{\phi\left(z, \tilde{Q}^{2}\right)}{z(z+\tilde{x}-1+i \epsilon)},
\end{gathered}
$$

and

$$
\begin{gathered}
N\left(\tilde{x}, Q_{T}^{2} / Q^{2}\right)=2\left\{(1-\tilde{x})^{2}\left[(\operatorname{Im} I(\tilde{x}))^{2}+(F+\operatorname{Re} I(\tilde{x}))^{2}\right]\right. \\
\left.+4 Q_{T}^{2} / Q^{2} \tilde{x}^{2} F^{2}+Q_{T}^{4} / Q^{4} \tilde{x}^{2} F^{2}\right\} .
\end{gathered}
$$

The variable $\tilde{x}$ acts to resolve the distribution amplitude much like the Bjorken variable resolves the structure functions,

$$
\tilde{x} \equiv \frac{x_{\bar{u}}}{1+Q_{T}^{2} / Q^{2}}=\frac{1}{2} \frac{x_{L}+\sqrt{x_{L}^{2}+4 s^{-1}\left(Q^{2}+Q_{T}^{2}\right)}}{1+Q_{T}^{2} / Q^{2}} .
$$

The factors $1 / z$ in eq. (10), (11) come from the gluon propagators and the factors $1 /(z+$ $\tilde{x}-1 \pm i \epsilon)$ arise from the quark propagator of Fig. 1b.

In contrast to Refs. [9] and [14] we did not omit terms $\mathcal{O}\left(\left(1-x_{\bar{u}}\right) Q_{T}^{2} / Q^{2}\right)$ and $\mathcal{O}\left(\left(1-x_{\bar{u}}\right)^{-1} Q_{T}^{4} / Q^{4}\right)$ and of higher orders. The nucleon distribution function $G_{q / N}$ does not appear in (7)-(9); thus only the pion distribution amplitude $\phi\left(z, \tilde{Q}^{2}\right)$ has to be specified. Before doing so we give some technical comments on our calculation.

We note that the internal quark line of Fig. 1b can go on-shell. The amplitude $M$ of equation (4), however, is always regular due to the $z$-integration [14] for realistic choices of $\phi\left(z, \tilde{Q}^{2}\right)$. This also can be read off from (11). The fact that the internal line goes on-shell does not cause a Sudakov suppression since our diagrams are the lowest order contribution of an inclusive process. In other words gluon emission to the final state will occur in the higher order corrections. Only when $x_{\bar{u}}$ approaches unity, where gluon emission is prohibited by kinematics, the Sudakov suppression will arise.

Our model and the parton model are not complementary, but rather different approximations to the Drell-Yan process. The diagrams of Fig. 1a,b give the whole leading order contribution in the specific kinematic region of large enough $x_{\bar{u}}, x_{\bar{u}}>0.5$ [9]. This is so because the gluon exchange is the resolution of the pion bound state and not a radiative correction. 
Now we can present our final results for $\lambda, \mu$ and $\nu$ for different choices of the pion distribution amplitude $\phi\left(z, \tilde{Q}^{2}\right)$. We find in general that the values of $\mu$ and $\nu$ are very sensitive to the choice of $\phi\left(z, \tilde{Q}^{2}\right)$ which we always take to be positive, symmetric, i.e. $\phi\left(z, \tilde{Q}^{2}\right)=\phi\left(1-z, \tilde{Q}^{2}\right)$, and normalized, $\int_{0}^{1} d z \phi\left(z, \tilde{Q}^{2}\right)=1$. Thus we will not restrict ourselves to the simplest case of $\phi(z) \sim \delta(z-1 / 2)$ considered in [9].

This sensitivity can be illustrated for the special case of $\tilde{x}=0.5$ for which $\operatorname{Re} I=$ $-2 F$. From eqs. (7)-(9) we get,

$$
\begin{aligned}
& \lambda\left(\tilde{x}=0.5, Q_{T}^{2} / Q^{2}\right)=\frac{1+4 \pi^{2} a^{2}-4 Q_{T}^{2} / Q^{2}+Q_{T}^{4} / Q^{4}}{1+4 \pi^{2} a^{2}+4 Q_{T}^{2} / Q^{2}+Q_{T}^{4} / Q^{4}}, \\
& \mu\left(\tilde{x}=0.5, Q_{T}^{2} / Q^{2}\right)=\frac{2 \sqrt{Q_{T}^{2} / Q^{2}}\left(1-Q_{T}^{2} / Q^{2}\right)}{1+4 \pi^{2} a^{2}+4 Q_{T}^{2} / Q^{2}+Q_{T}^{4} / Q^{4}}, \\
& \nu\left(\tilde{x}=0.5, Q_{T}^{2} / Q^{2}\right)=\frac{4 Q_{T}^{2} / Q^{2}}{1+4 \pi^{2} a^{2}+4 Q_{T}^{2} / Q^{2}+Q_{T}^{4} / Q^{4}},
\end{aligned}
$$

where $a \equiv \phi(z=0.5) / F$.

From these formulas we see that $\mu$ and $\nu$ in this case are not suppressed only if $a$ is a sufficiently small number. This is true for the so-called two-humped distribution amplitude [15] which has a dip around $z=0.5$. On the other hand, the choice of a convex distribution amplitude, e.g. the asymptotic one, $\phi(z)=6 z(1-z)$, will always produce suppressed $\mu$ and $\nu$ at $\tilde{x}=0.5$.

We return now to our general results, eqs. (7)-(9). In Fig. 2 we plot $\lambda, \mu, \nu$ and $2 \nu-(1-\lambda)$ versus $x_{\bar{u}}$ for $\sqrt{Q_{T}^{2} / Q^{2}}=0.25$ for different choices of $\phi\left(z, \tilde{Q}^{2}\right)$ together with the data of Ref. [5]. For the two-humped distribution amplitude we have chosen the evolution parameter $\tilde{Q}^{2}$ to be effectively $\sim 4 \mathrm{GeV}^{2}$. The solid line is the result for the two-humped $\phi(z)$ where powers of $\left(Q_{T}^{2} / Q^{2}\right)^{n / 2}$ were dropped for $n \geq 3$ in eqs. (7)-(9). We note that corrections to our model may induce such terms, thus the difference between the dashed and the solid lines should be viewed as the uncertainty of our predictions. We also show the data points of Ref. [5] averaged in the intervals $4.05<\sqrt{Q^{2}}<8.55 \mathrm{GeV}$ and $0<\sqrt{Q_{T}^{2}}<5 \mathrm{GeV}$.

In Fig. 3 the same quantities are shown versus $\sqrt{Q_{T}^{2}}$ for $x_{\bar{u}}=0.6$ and $\sqrt{Q^{2}}=6 \mathrm{GeV}$. The data points in this case are averaged over intervals $4.05<\sqrt{Q^{2}}<8.55 \mathrm{GeV}$ and $0.2<x_{\bar{u}}<1$ and taken from Ref. [5]. All the data points were averaged over the intervals 
defined above in Ref. [5]. We would rather prefer to use the unaveraged data which are not available. The use of the averaged over $x_{\bar{u}}$ data in Fig. 3 forced us to fix the value $x_{\bar{u}}=0.6$ for our theoretical prediction which is rather low for our model and pushes it to the limits of its applicability.

Finally we would like to comment on the limitations of our model and corrections to it. The bound state effects considered here should have received a truly non-perturbative treatment. We have restricted ourselves to a perturbative approximation to the problem. This approximation makes sense only at large enough $x$ which we have chosen to be $>0.5$. The contribution of more than one hard gluon exchange will be suppressed by powers of $\alpha_{s}$ in this case. The contribution of soft gluons to the pion bound state is taken into account in the evolution of the distribution amplitude. The higher Fock states of the pion are expected to be suppressed when $x$ is large enough [12]. The pion and the nucleon are not treated symmetrically in our model, namely the nucleon bound state effects are not taken into account since in the kinematic region we consider, $x_{\bar{u}}$ is always large and $x_{u}$ is always small. Gluon emission to the final state will first contribute to the evolution of the parton distribution functions. Hard gluon emission is suppressed by powers of $\alpha_{s}$. No attempt of a systematic inclusion of higher order or mass effects was made.

The coefficient functions $\lambda, \mu$, and $\nu$ at large $x>0.5$ are very sensitive to the shape of the projectile's distribution amplitude $\phi\left(z, \tilde{Q}^{2}\right)$, the basic hadron wavefunction which describes the distribution of light-cone momentum fractions in the lowest-particle number valence Fock state. Measurements of meson form factors [12] and other exclusive and semiexclusive processes [16] at large momentum transfer can only provide global constraints on the shape of $\phi\left(z, \tilde{Q}^{2}\right)$; in contrast, the angular dependence of the lepton pair distributions can be used to provide local measurements of the shapes of these hadron wavefunctions. Detailed measurements of the angular distribution of leptons as a function of both $x$ and $Q_{T}$ for the reactions $H p \rightarrow \ell^{+} \ell^{-} X$ for the whole range of fixed target beams $H=\pi, K, \bar{p}, p$, and $n$ will open up a new window on the structure of hadrons at the amplitude level.

Our analysis shows that the broad, two-humped, distribution amplitude for the pion which was obtained within the context of QCD sum rules [15] can account for the main features of the data. In contrast, narrow momentum distributions, characteristic of weak 
hadronic binding, predict the wrong sign for the observed azimuthal angular coefficients $\mu$ and $\nu$.

\section{Acknowledgments}

We wish to thank V. Telegdi for conversations regarding the experimental data, particularly the fact that the observed increase of the variable $\nu$ with the dimuon transverse momentum is at variance with perturbative QCD calculations. We are grateful to Yu. L. Dokshitzer, O. Nachtmann and W. J. Stirling for useful suggestions and comments on the manuscript. We also benefited from discussions with H. Anlauf, V. M. Braun, T. Hyer, V. A. Khoze, and M. Peskin. 


\section{References}

[1] K. Freudenreich, Int. J. Mod. Phys. A 5(1990) 3643; Yu.L. Dokshitzer, D.I. Dyakonov and S.I. Troyan, Phys. Rep. 58 (1980) 269; Proceedings of the Workshop on Drell-Yan Processes, Fermilab, Batavia, 1982

[2] C.S. Lam and W.K. Tung, Phys. Rev. D 21 (1980) 2712

[3] P. Chiappetta and M. Le Bellac, Z. Phys. C 32 (1986) 521

[4] NA10 Collab. S. Falciano et al., Z. Phys. C 31 (1986) 513; NA10 Collab. M. Guanziroli et al., Z. Phys. C 37 (1988) 545

[5] J.S. Conway et al., Phys. Rev. D 39 (1989) 92

[6] J.G. Heinrich et al., Phys. Rev. D 44 (1991) 44

[7] A. Brandenburg, O. Nachtmann and E. Mirkes, Z. Phys. C 60 (1993) 697

[8] E.L. Berger and S.J. Brodsky, Phys. Rev. Lett. 42 (1979) 940; S.J. Brodsky, E.L. Berger and G.P. Lepage in Proceedings of the Workshop on Drell-Yan Processes, Fermilab, Batavia, 1982, p. 187

[9] E.L. Berger, Z. Phys. C 4 (1980) 289

[10] S.D. Drell and T.M. Yan, Phys. Rev. Lett. 25 (1970) 316

[11] G. Altarelli, R.K. Ellis, M. Greco and G. Martinelli, Nucl. Phys.B 246 (1984) 12

[12] G.P. Lepage and S.J. Brodsky, Phys. Rev. D 22 (1980) 2157

[13] S.J. Brodsky, P. Hoyer, A.H. Mueller and W.K. Tang, Nucl. Phys. B 369 (1992) 519; K. Eshola, P. Hoyer, M. Vanttinen, R. Vogt (to be published)

[14] S. Matsuda, Phys. Lett. B 119 (1982) 207

[15] V.L. Chernyak and A.R. Zhitnitsky, Phys. Rep. 112 (1984) 173

[16] A.V. Efremov and A.V. Radyushkin, Phys. Lett. B 94 (1980) 245; M.K. Chase, Nucl. Phys. B 167 (1980) 125; S.J. Brodsky and G.P. Lepage, Phys. Rev. D 24 (1981) 1808; E. Braaten, Phys. Rev. D 28 (1983) 524; E. Maina and G. Farrar, Phys. Lett. B 206 (1988) 120; T. Hyer, Phys. Rev. D 48 (1993) 147 


\section{Figure Captions}

Fig. 1: Diagrams (a) and (b) give the leading contribution to the amplitude of reaction (4). Diagram (c) gives a typical (one out of four) contribution to the cross section (6).

Fig. 2: The angular distribution coefficients $\lambda, \mu$ and $\nu$ and the Lam-Tung combination, $2 \nu-(1-\lambda)$, in the Gottfried-Jackson frame, versus $x_{\bar{u}}$ for $\sqrt{Q_{T}^{2} / Q^{2}}=0.25$. The dotted line corresponds to $\phi(z)=\delta(z-1 / 2)$, the dashed-dotted line corresponds to the asymptotic $\phi(z)=6 z(1-z)$ and the dashed line shows the results for the two humped distribution amplitude, $\phi(z)=26 z(1-z)(1-50 / 13 z(1-z))$. The solid line is the result for the two-humped $\phi(z)$ where powers of $\left(Q_{T}^{2} / Q^{2}\right)^{n / 2}$ were dropped for $n \geq 3$ in eqs. (13)-(15). The data points (averaged as explained in the text) are taken from Ref. [5].

Fig. 3: The same quantities as in Fig. 2 are shown. versus $\sqrt{Q_{T}^{2}}$ for $x_{\bar{u}}=0.6$ and $\sqrt{Q^{2}}=6 \mathrm{GeV}$. 
This figure "fig1-1.png" is available in "png" format from: http://arxiv.org/ps/hep-ph/9403361v2 
This figure "fig2-1.png" is available in "png" format from: http://arxiv.org/ps/hep-ph/9403361v2 
This figure "fig3-1.png" is available in "png" format from: http://arxiv.org/ps/hep-ph/9403361v2 